\title{
On-Line Learning of Rectangles and Unions of Rectangles
}

\author{
ZHIXIANG CHEN \\ Department of Computer Science, Boston University, Boston, MA 02215, USA
}

zchen@cs.bu.edu.

WOLFGANG MAASS

maass@igi.tu-graz.ac.at Institute for Theoretical Computer Science, Technische Universitaet Graz, Klosterwiesgasse 32, A-8010 Graz, Austria

\section{Editor: Lisa Hellerstein}

\begin{abstract}
We design efficient algorithms for on-line learning of axis-parallel rectangles (and for the union of two such rectangles) in the common model for on-line learning with equivalence queries. With regard to the learning of rectangles in arbitrary dimensions $d$ we solve the following open problem:

Is there an algorithm for on-line learning of rectangles $\prod_{i=1}^{d}\left\{a_{i}, a_{i}+1, \ldots, b_{i}\right\}$ over a discrete domain $\{1, \ldots, n\}^{d}$ whose error bound is polylogarithmic in the size $n^{d}$ of the domain (i.e. polynomial in $d$ and $\log n$ )?

We give a positive solution by introducing a new design technique that appears to be of some interest on its own. The new learning algorithm for rectangles consists of $2 d$ separate search strategies that search for the parameters $a_{1}, b_{1}, \ldots, a_{d}, b_{d}$ of the target rectangle. A learning algorithm with this type of modular design tends to fail because of the well known "credit assignment problem": Which of the $2 d$ local search strategies should be "blamed" when the global algorithm makes an error? We propose here a rather radical solution to this problem: each local search strategy that is possibly involved in an error of the global algorithm will be blamed. With this radical solution it is unavoidable that frequently local search strategies will be blamed incorrectly. We overcome this difficulty by employing local search strategies ("error tolerant binary search") that are able to tolerate such incorrect credit assignments. The structure of this learning algorithm is reminiscent of "finite injury priority constructions" in recursive function theory.

Section 4 contains another application of this design technique: an algorithm for learning the union of two rectangles in the plane.
\end{abstract}

Keywords: On-line learning, computational learning theory, geometrical learning problems, finite injury priority constructions

\section{Introduction}

In our first result we consider the concept class

$$
B O X_{n}^{d}:=\{\phi\} \cup\left\{\prod_{i=1}^{d}\left\{a_{i}, \ldots, b_{i}\right\} \mid 1 \leq a_{i} \leq b_{i} \leq n \quad \text { for } i=1, \ldots, d\right\}
$$

over the domain $\{1, \ldots, n\}^{d}$. We will refer to the elements of $B O X_{n}^{d}$ as boxes, or equivalently as rectangles.

Our learning model is the standard model for on-line learning (see Angluin, 1988; Littlestone, 1987; Maass \& Turán, 1992). A learning process for a concept class $\mathcal{C}$ over a domain $X$ is viewed as a dialogue between a learner $A$ and the environment. The goal of the learner $A$ is to "learn" an unknown target concept $C_{T} \in \mathcal{C}$ that has been fixed by the 
environment. In order to gain information about $C_{T}$ the learner proposes hypotheses $H$ from a fixed hypothesis space $\mathcal{H}$ with $\mathcal{C} \subseteq \mathcal{H} \subseteq 2^{X}$.

Whenever $H \neq C_{T}$ for the proposed hypothesis $H$, the environment responds with some counterexample (CE) $g \in H \Delta C_{T}:=\left(C_{T}-H\right) \cup\left(H-C_{T}\right) . g$ is called a positive counterexample (PCE) if $g \in C_{T}-H$, and $g$ is called a negative counterexample (NCE) if $g \in H-C_{T}$. Each new hypothesis $H_{s+1}$ of the learner (resp. learning algorithm) $A$ may depend on the earlier hypotheses $H_{1}, \ldots, H_{s}$ and the given counterexamples $g_{j} \in H_{j} \Delta C_{T}$ for $j=1, \ldots, s$.

One defines the resulting learning complexity of a learning algorithm $A$ by

$$
\begin{aligned}
L C(A):=\max \{s \in \mathrm{N} \mid & \text { there is some } C_{T} \in \mathcal{C} \text { and a sequence } g_{1}, \ldots, g_{s-1} \text { of } \\
& \text { counterexamples to the hypotheses } H_{1}, \ldots, H_{s-1} \text { of the } \\
& \text { learner } \left.A \text { such that } H_{s} \neq C_{T}\right\} .
\end{aligned}
$$

The learning complexity of concept class $\mathcal{C}$ with hypothesis space $\mathcal{H}$ is defined by

$$
\begin{gathered}
L C^{\mathcal{H}}(\mathcal{C}):=\min \{L C(A) \mid A \text { is a learning algorithm for } \mathcal{C} \text { with hypothesis space } \\
\mathcal{H}\} .
\end{gathered}
$$

One sets $L C(\mathcal{C}):=L C^{\mathcal{C}}(\mathcal{C})$ and $L C-A R B(\mathcal{C}):=L C^{2^{x}}(\mathcal{C})$.

This learning model is equivalent to Littlestone's model (1987) for "mistake bounded on-line learning". In Littlestone's model, which is somewhat closer to realistic learning situations, the learner proposes at each step $s$ some hypothesis $H_{s} \in \mathcal{H}$. He then uses this hypothesis $H_{s}$ to "predict" the label $y_{s}$ of the example $\left\langle x_{s}, y_{s}\right\rangle \in X \times\{0,1\}$ (with $\left.y_{s}=C_{T}\left(x_{s}\right)\right)$ that is given without the label $y_{s}$ to the learner at step $s$. Whenever this prediction is incorrect, one says that the learner has made a mistake at step $s$. The goal of the learner is to minimize the total number of mistakes that he makes. It is easy to show (see Littlestone, 1987) that the worst case mistake bound of the best learning algorithm in this model is equal to $L C^{\mathcal{H}}(\mathcal{C})$. This holds no matter whether the learner is allowed to revise his hypothesis at every step, or only at those steps where he has made a mistake.

It is known that $L C\left(B O X_{n}^{d}\right) \geq L C-A R B\left(B O X_{n}^{d}\right)=\Theta(d \log n)$. The upper bound $O(d \log n)$ for $L C-A R B\left(B O X_{n}^{d}\right)$ follows by considering the HALVING-algorithm (see Angluin, 1988; Littlestone, 1987; Maass \& Turán, 1992). The lower bound $\Omega(d \log n)$ is shown by constructing a decision tree for $B O X_{n}^{d}$ in which every leaf has depth $\Omega(d \log n)$. This is sufficient by a result of Littlestone (1987) (see also Maass \& Turán, 1992).

The HALVING-algorithm uses arbitrary subsets of the domain as hypotheses. With regard to learning algorithms for $B O X_{n}^{d}$ that use computationally feasible hypotheses there exist two quite different approaches. Both of these algorithms use hypotheses from $B O X_{n}^{d}$. There is a learning algorithm $B$ with $L C(B)=O(d \cdot n)$ that issues as its next hypothesis always the smallest $C \in B O X_{n}^{d}$ that is consistent with all preceding counterexamples. This algorithm is frequently considered in the special case $n=2$ where the concepts $C \in B O X_{2}^{d}$ correspond to monomials over $d$ boolean variables (see the algorithm for the complementary class 1-CNF in Valiant, 1984).

It is less trivial (even for $d=2$ ) to design a learning algorithm $D$ for $B O X_{n}^{d}$ with computationally feasible hypotheses such that $L C(D)=O(f(d) \log n)$ for some function 
$f: \mathbf{N} \rightarrow \mathbf{N}$. An algorithm $D$ of this type (which uses hypotheses from $B O X_{n}^{d}$ ) was exhibited in Maass \& Turán $(1989,1994)$. However this algorithm $D$ learns separately each of the $2^{d}$ corners of the target concept, and hence $L C(D)$ is exponential in $d$ (i.e. $\left.f\left(a^{\prime}\right) \geq 2^{d}\right)$.

The question whether the advantageous features of both learning algorithms $B$ and $D$ can be combined in a single algorithm $S$ with $L C(S) \leq \operatorname{poly}(d, \log n)$ was first brought to our attention by David Haussler (1989; see also Maass \& Turán, 1994).

A learning algorithm $S$ which achieves this performance is exhibited in section 3 of this paper. It proceeds in a completely different way than the two previously described learning algorithms for $B O X_{n}^{d}$. We describe the main component of the new algorithm in section 2 .

The main ingredient of this learning algorithm is a novel solution of the "credit assignment problem". The creditassignment problem may be defined as "the problem of assigning credit or blame to the individual decisions that led to some overall result" (Cohen \& Feigenbaum, 1982). Obviously this problem is ubiquitous not just in Artificial Intelligence, but also in the study of adaptive neural networks, where credit or blame for the overall performance of the network has to be distributed to the individual components of the network.

The credit assignment problem in the case of on-line learning of rectangles is the following. When the learner receives a negative counterexample $\left\langle x_{1}, \ldots, x_{d}\right\rangle$ to his current hypothesis $\prod_{i=1}^{d}\left\{a_{i}, \ldots, b_{i}\right\}$, it is clear that the learner has to change at least one of the intervals $\left\{a_{i}, \ldots, b_{i}\right\}$ so that it no longer contains $x_{i}$. But it is not clear which of the intervals $\left\{a_{i}, \ldots, b_{i}\right\}$ should be changed.

Our new learning algorithm for rectangles consists of $2 d$ separate search strategies that search for the $2 d$ endpoints $a_{1}^{T}, b_{1}^{T}, \ldots, a_{d}^{T}, b_{d}^{T}$ of the $d$ intervals $\left\{a_{i}^{T}, \ldots, b_{i}^{T}\right\}$ of the targetrectangle $\prod_{i=1}^{d}\left\{a_{i}^{T}, \ldots, b_{i}^{T}\right\}$. The main problem is which of the $2 d$ local search strategies should be "blamed" when the global algorithm makes an error? We propose here a rather radical solution to this credit assignment problem: each local search strategy that is possibly involved in an error of the global algorithm will be blamed. With this radical solution it is unavoidable that frequently local search strategies will be blamed incorrectly. We overcome this difficulty by employing local search strategies ("error tolerant binary search") that are able to tolerate such incorrect credit assignments.

Our solution of the credit assignment problem is quite reminiscent of a famous construction method in recursive function theory, the socalled finite injury priority construction (see Soare, 1987). This linkage is of some methodological interest insofar as priority arguments with injuries are the dominant design technique in recursive function theory, but so far there are hardly any applications of this technique for the design of concrete algorithms in computer science.

Section 4 contains another application of this design technique: an algorithm for learning the union of two rectangles in the plane. We assume here that the learner knows already that the top left corner of the domain is contained in one rectangle, and the bottom right corner in the other. Nevertheless this learning problem is substantially more complicated than the preceding one: The obvious local search procedures that search for the lengths of the sides of the two rectangles are likely to get not only false negative counterexamples (as in the preceding learning problem), but also false positive counterexamples. This complication arises from the fact that in general the learner does not know to which one of the two 
rectangles of $C_{T}$ a positive counterexample belongs. Nevertheless one can construct for this learning problem an efficient learning algorithm whose learning complexity is asymptotically optimal. Again this algorithm consists of suitable versions of binary search as modules, which will tolerate certain incorrect credit assignments.

This positive result for learning the union of two rectangles provides a contrast to earlier results about efficiently learnable concept classes $\mathcal{C}$ such as halfplanes over $\{1, \ldots, n\}^{2}$, or monomials, for which one has shown that $\mathcal{U}-2-\mathcal{C}:=\left\{C_{1} \cup C_{2} \mid C_{1}, C_{2} \in \mathcal{C}\right\}$ is not efficiently learnable (see Maass \& Turán, 1994; Pitt \& Valiant, 1988).

\section{An algorithm for binary search that tolerates one-sided errors}

In this section we consider an extension of the notion of a "negative counterexample", and along with it an extension of the previously described learning model.

Assume $C_{T} \in \mathcal{C}$ is the target concept and $H_{s}$ is the current hypothesis of the learner. The environment may respond in the extended model with a positive counterexample ("PCE") $g \in C_{T}-H_{s}$, with a true negative counterexample ("true NCE") $g \in H_{s}-C_{T}$, or with a false negative counterexample ("false NCE") $g \in H_{s} \cap C_{T}$. Note that the environment is allowed to respond with a false NCE even if $H_{s}=C_{T}$. We extend the notion of a negative counterexample (NCE) so that it subsumes both true and false NCE's. The environment is not required to tell the learner to which of these categories a counterexample $g$ belongs.

We define a binary search algorithm $T B S_{n}$ (the "T" stands for error-tolerant) for learning the "head" $h$ of a halfinterval $\{1, \ldots, h\} \subseteq\{1, \ldots, n\}$ in this extended learning model. The new algorithm $S$ for learning rectangles $C_{T}=\prod_{i=1}^{d}\left\{a_{i}, \ldots, b_{i}\right\} \in B O X_{n}^{d}$ (see section 3) will consist of $2 d$ separate copies of the here defined error-tolerant binary search algorithm $T B S$ : in each dimension $i$ it uses separate copies of $T B S$ and its symmetric counterpart $T B S^{*}$ for learning the "head" $b_{i}$ and the "tail" $a_{i}$ of the interval $\left\{a_{i}, \ldots, b_{i}\right\}$. Although this learning algorithm $S$ for $B O X_{n}^{d}$ will receive only true counterexamples, the individual binary search procedures may also receive false negative counterexamples. This is a consequence of our quite radical solution to the associated "credit assignment problem", where we blame each of the $2 d$ subroutines for binary search for any error of the learning algorithm $S$. In particular a true NCE for $S$ will result in a true NCE for at least one subroutine and false NCE's for up to $d-1$ other subroutines.

In this section we consider the concept class

$$
H E A D_{n}:=\{\{1, \ldots, j\} \mid j \in\{1, \ldots, n\}\}
$$

over the domain $\{1,2, \ldots, n\}$.

At the beginning of each step $r$ of a learning process in the extended learning model the learner issues a hypothesis $H_{r}:=\left\{1, \ldots, h_{r}\right\} \in H E A D_{n}$. If $H_{r} \neq C_{T}$, then the learner will receive at step $r$ the counterexample $g_{r} \in\{1, \ldots, n\}$. We set

$$
\begin{aligned}
p_{s}:=\max \left(\{1\} \cup\left\{g_{r} \mid 1 \leq r \leq s \text { and } g_{r} \text { was a PCE }\right\}\right) \\
n_{s}:=\min \left(\{ n + 1 \} \cup \left\{g_{r} \mid 1 \leq r \leq s, g_{r}>p_{s},\right.\right. \\
\left.\left.\quad \text { and } g_{r} \text { was a (true or false) NCE }\right\}\right) \\
n_{s}^{\text {true }}:=\min \left(\{n+1\} \cup\left\{g_{r} \mid 1 \leq r \leq s \text { and } g_{r} \text { was a true NCE }\right\}\right) .
\end{aligned}
$$




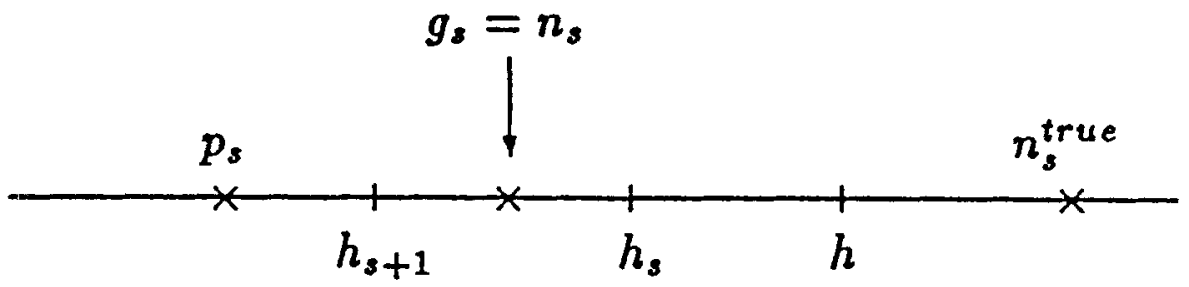

Figure 1. A typical scenario in the procedure $T B S_{n}$, where $\{1, \ldots, h\}$ is the target concept and the hypothesis $\left\{1, \ldots, h_{s}\right\}$ is "refuted" at step $s$ by a false NCE $g_{s}$.

The interpretation of these parameters is quite obvious. $p_{s}$ is the largest PCE received by step $s$, and $n_{s}^{\text {true }}$ is the smallest true NCE received by step $s$. Hence it is clear that the endpoint $h$ of the target concept $H_{T}=\{1, \ldots, h\} \in H E A D_{n}$ lies in the "undetermined interval" $\left\{p_{s}, \ldots, n_{s}^{\text {true }}-1\right\}$. Unfortunately the learner does in general not know the value of $n_{s}^{\text {true }}$ at step $s$. His best possible approximation to $n_{s}^{\text {true }}$ is provided by the parameter $n_{s}$, which is the minimum of all those NCE's received by step $s$ which have not yet been shown to be false NCE's by the end of step $s$.

The following search algorithm $\mathrm{TBS}_{n}$ (where TBS stands for error-tolerant binary search) provides the basic module for our learning algorithm for rectangles.

\section{Definition of the binary search algorithm $T B S_{n}$ for learning $H E A D_{n}$ in the extended learning model:}

The algorithm $T B S_{n}$ issues at step $s$ the hypothesis $\left\{1, \ldots, h_{s}\right\}$.

Set $h_{1}:=1$.

For $s \geq 1$ set $h_{s+1}:=h_{s}$ if $g_{s}$ is a NCE and $g_{s} \leq p_{s}$.

[If $g_{s} \leq p_{s}$ it is clear that $g_{s}$ is a false NCE, hence it can safely be ignored.]

Else, we define

$$
h_{s+1}:= \begin{cases}\min \left(\left\{n_{s}-1\right\} \cup\left\{h_{r} \mid 1 \leq r \leq s \text { and } p_{s} \leq h_{r}<n_{s}\right\}\right), \\ & \text { if } g_{s} \text { is a PCE. } \\ p_{s}+\left\lfloor\frac{n_{s}-p_{s}}{2}\right\rfloor, & \text { if } g_{s} \text { is a (true or false) NCE. }\end{cases}
$$

In this algorithm $T B S_{n}$ the hypothesis $\left\{1, \ldots, h_{s+1}\right\}$ at step $s+1$ is defined as in the usual binary search procedure in the case where $g_{s}$ is a NCE which is not obviously false at step $s$ (i.e. $g_{s}>p_{s}$ ). A typical situation of this type is illustrated in Figure 1 .

As a consequence of this clause in the definition of $T B S_{n}$ one can show in Lemma 2.3 that any true NCE reduces the length of the "undetermined interval" $\left\{p_{s}, \ldots, n_{s}^{\text {true }}-1\right\}$ by at least $50 \%$ (as in the usual binary search procedure). The strategy of the quite different definition of the hypotheses $\left\{1, \ldots, h_{s+1}\right\}$ after a PCE at step $s$ is to treat PCE's as a particularly valuable resource. This is justified, since PCE's are always true counterexamples. Therefore, instead of halving the open interval in an upwards direction, one 
moves the hypothesis direct up to the least unrefuted NCE, respectively the least unrefuted earlier hypothesis. The justification for this clause in the definition is given in Lemma 2.4. It is shown there that a second PCE after this move will bring definite progress: it either unmasks a false NCE, or it definitely refutes an earlier hypothesis that was previously only "refuted" by false NCE's.

Note that the more familiar halving of the open interval in an upwards direction after each PCE (as in the regular binary search procedure) uses PCE's in a less economic fashion (if false NCE's are present). It could then occur that one uses a sequence of $\log n / 2$ PCE's just to find out that one earlier NCE (to which these PCE's converge from below) was a false NCE.

THEOREM 2.1 Assume that f false NCE's occur in a learning process for $H E A D_{n}$ with learning algorithm $T B S_{n}$. Then at most $\log n$ true NCE's and at most $\log n+3 f+1$ PCE's occur in this learning process.

In order to prove Theorem 2.1 we analyze the properties of algorithm $T B S_{n}$ in three simple Lemmata.

\section{LEMMA 2.2}

a) $p_{s}+\left\lfloor\frac{n_{s}-p_{s}}{2}\right\rfloor \leq n_{s}-1$ for all $s \geq 1$.

b) $p_{s} \leq h_{s+1} \leq n_{s}-1$ for all $s \geq 1$.

c) If $g_{s}$ is a (true or false) NCE, then $h_{s+1} \leq h_{s}$.

\section{Proof:}

a) Note that $p_{s}<n_{s}$ by the definition of $n_{s}$.

b) This follows from part a).

c) If $g_{s} \leq p_{s}$ then $h_{s+1}=h_{s}$. Assume that $g_{s}>p_{s}$ and $s>1$. Since $h_{s} \leq n_{s-1}-1$ by b), we have $n_{s}=g_{s} \leq h_{s}$. Furthermore $h_{s+1}=p_{s}+\left\lfloor\frac{n_{s}-p_{s}}{2}\right\rfloor \leq n_{s}$ (see part a)).

LEMMA 2.3 Assume $r<s$ and $g_{r}, g_{s}$ are true NCE's. Then

$$
n_{s}^{\text {true }}-p_{s} \leq \frac{n_{r}^{\text {true }}-p_{r}}{2}
$$

Proof: It is obvious that $r>1$. Thus $n_{r}^{\text {true }}=n_{r}=g_{r}$, since $p_{r}<g_{r} \leq h_{r} \leq n_{r-1}-1$ by Lemma $2.2 \mathrm{~b}$ ). One shows in the same way that $n_{s}^{\text {true }}=n_{s}=g_{s}$.

Since $g_{r}$ is a NCE one has $h_{r+1}=p_{r}+\left\lfloor\frac{n_{r}^{\text {true }}-p_{r}}{2}\right\rfloor$. 
Case 1: $p_{s}>h_{r+1}$

Then $p_{s} \geq p_{r}+\left\lfloor\frac{n_{r}^{\text {true }}-p_{r}}{2}\right\rfloor+1 \geq \frac{p_{r}+n_{r}^{\text {true }}}{2}$, hence $n_{s}^{\text {true }}-p_{s} \leq n_{r}^{\text {true }}-p_{s} \leq n_{r}^{\text {true }}-$ $\left(\frac{p_{r}+n_{r}^{\text {true }}}{2}\right)=\frac{n_{r}^{\text {true }}-p_{r}}{2}$.

Case 2: $p_{s} \leq h_{r+1}$

Then $g_{j} \leq h_{r+1}$ for every $j \in\{r+1, \ldots, s-1\}$ such that $g_{j}$ is a PCE. Hence $h_{j+1} \leq h_{r+1}$ for each such $j$ (by the definition of $h_{j+1}$ ). Together with Lemma $2.2 \mathrm{c}$ ) this implies that $h_{j+1} \leq h_{r+1}$ for every $j \in\{r+1, \ldots, s-1\}$. In particular we have shown that $h_{s} \leq h_{r+1}$. Thus $n_{s}^{\text {true }}=g_{s} \leq h_{r+1}$. Therefore $n_{s}^{\text {true }}-p_{s} \leq h_{r+1}-g_{s} \leq p_{r}+\left(\frac{n_{r}^{\text {true }}-p_{r}}{2}\right)-p_{s} \leq$ $p_{r}+\left(\frac{n_{r}^{\text {true }}-p_{r}}{2}\right)-p_{r}=\frac{n_{r}^{\text {true }}-p_{r}}{2}$.

LEMMA 2.4 Assume that $g_{s}$ and $g_{s+1}$ are PCE's. Then $g_{s+1} \geq n_{s}$, or $g_{s+1}>h_{r}$ for some $r \in\{1, \ldots, s\}$ with $p_{s} \leq h_{r}<n_{s}$. In other words: $g_{s+1}$ proves that an earlier $N C E$ was a false NCE, or it definitely refutes an earlier hypothesis $\left\{1, \ldots, h_{r}\right\}$ which had received a counterexample at step $r$, but which was at step $s$ consistent with all currently unrefuted counterexamples.

Proof: By construction one has $g_{s+1}>h_{s+1}=\min \left(\left\{n_{s}-1\right\} \cup\left\{h_{r} \mid 1 \leq r \leq s\right.\right.$ and $\left.p_{s} \lessgtr h_{r}<n_{s}\right\}$ ).

Proof of Theorem 2.1: Lemma 2.3 implies that at most $\log n$ true NCE's occur in any learning process with algorithm $T B S_{n}$. Hence at most $\log n+f$ NCE's occur in the considered learning process $Q$. Thus there exist at most $\log n+f+1$ maximal blocks of successive PCE's in this learning process $Q$. Consider any such maximal block $B$ that consists of $k+1$ PCE's $g_{s}, \ldots, g_{s+k}$. Set

$$
\begin{aligned}
k_{1}^{B}:= & \mid\left\{j \mid j \in\{s+1, \ldots, s+k\} \text { and } g_{j} \geq n_{j-1}\right\} \mid \\
k_{2}^{B}:= & \mid\left\{j \mid j \in\{s+1, \ldots, s+k\} \text { and } g_{j}>h_{r} \text { for some } r \in\{1, \ldots, j-1\}\right. \\
& \text { with } \left.p_{j-1} \leq h_{r}<n_{j-1}\right\} \mid .
\end{aligned}
$$

By Lemma 2.4 we have $k_{1}^{B}+k_{2}^{B} \geq k$.

Each time when $g_{j} \geq n_{j-1}$ (as in the definition of $k_{1}^{B}$ ), then an earlier NCE gets proven false at step $j$. This happens at most once for each of the $f$ false NCE's.

Each time when $g_{j}$ provides a counterexample to an earlier hypothesis $\left\{1, \ldots, h_{r}\right\}$ that was consistent with all unrefuted counterexamples at the beginning of step $j$ (as in the definition of $k_{2}^{B}$ ), then this hypothesis $\left\{1, \ldots, h_{r}\right\}$ can never appear to be consistent again at a later step $t$ (since $p_{t} \geq g_{j}>h_{r}$ ). Furthermore this event can only occur if the original counterexample $g_{r}$ to $\left\{1, \ldots, h_{r}\right\}$ was a false NCE. Thus altogether there are only $f$ hypotheses $\left\{1, \ldots, h_{r}\right\}$ for which this event can ever occur. 
Thus we have shown that $\sum\left\{k_{1}^{B} \mid B\right.$ is a maximal block of PCE's in $\left.Q\right\} \leq f$ and $\sum\left\{k_{2}^{B} \mid B\right.$ is a maximal block of PCE's in $\left.Q\right\} \leq f$. Altogether we have shown that at most $\log n+3 f+1$ PCE's occur in the considered learning process $Q$.

Remark 2.5 One can construct in the same manner a learning algorithm $T B S_{n}^{*}$ for the concept class

$$
T A I L_{n}:=\{\{j, j+1, \ldots, n\} \mid 1 \leq j \leq n\}
$$

that satisfies an analogous version of Theorem 2.1 .

Remark 2.6 There exist already various algorithms for binary search in the presence of two-sided errors, see e.g. Dhagat, Gacs, \& Winkler (1992) and Borgstrom \& Kosaraju (1993). These algorithms do not provide sufficiently strong bounds (e.g. on the number of true NCE's) to be useful for our application in section 3.

\section{A learning algorithm for $B O X_{n}^{d}$ whose error bound is polynomial in $d$ and $\log n$}

\section{ThEOREM $3.1 L C\left(B O X_{n}^{d}\right)=O\left(d^{2} \log n\right)$.}

Proof: Consider any target concept $C_{T}=\prod_{i=1}^{d}\left\{a_{i}, \ldots, b_{i}\right\} \in B O X_{n}^{d}$. The learning algorithm $S$ for $B O X_{n}^{d}$ issues $H_{1}:=\emptyset$ as its first hypothesis. If $H_{1} \neq C_{T}$ then $S$ receives a PCE $u=\left\langle u_{1}, \ldots, u_{d}\right\rangle \in C_{T}$. Henceforth the algorithm $S$ splits the task of learning $C_{T}$ into $2 d$ separate subtasks: The learning of $\left\{u_{i}, \ldots, b_{i}\right\} \subseteq\left\{u_{i}, \ldots, n\right\}$ (i.e. of a concept from $H E A D_{n-u_{i}+1}$ over the transformed domain $\left\{u_{i}, \ldots, n\right\}$ ) and the learning of $\left\{a_{i}, \ldots, u_{i}\right\} \subseteq\left\{1, \ldots, u_{i}\right\}$ (i.e. of a concept from $T A I L_{u_{i}}$ ) for $i=1, \ldots, d$. For each $i \in\{1, \ldots, d\}$ the algorithm $S$ employs $T B S_{n-u_{i}+1}$ for the former and $T B S_{u_{i}}^{*}$ for the latter subtask.

One sets $H_{2}:=\{u\}$. Assume that at any step $r \geq 2$ the learning algorithm $S$ for $B O X_{n}^{d}$ has issued a hypothesis $H_{r}:=\prod_{i=1}^{d}\left\{h_{i}^{*}, \ldots, h_{i}\right\}$. Then the next hypothesis $H_{r+1}$ is determined in the following way by the $2 d$ subroutines.

Let $x=\left\langle x_{1}, \ldots, x_{d}\right\rangle \in C_{T} \Delta H_{r}$ be the counterexample to the hypothesis $H_{r}$ of algorithm $S$. Note that we use the notion of a counterexample for algorithm $S$ in the traditional sense (i.e. $x$ is a PCE or a true NCE). If $x$ is a PCE to hypothesis $H_{r}$, then for at least one $i \in\{1, \ldots, d\}$ the point $x_{i}$ is a PCE to the current hypothesis of one of the two subroutines $T B S_{n-u_{i}+1}$ or $T B S_{u_{i}}^{*}$. For each such $i$ one changes the interval in the $i$-th dimension according to the next hypothesis of the subroutine $T B S_{n-u_{i}+1}$ resp. $T B S_{u_{i}}^{*}$. For other $i$ one has $x_{i} \in\left\{h_{i}^{*}, \ldots, h_{i}\right\}$, and one repeats in these dimensions the same interval $\left\{h_{i}^{*}, \ldots, h_{i}\right\}$ in the next hypothesis $H_{r+1}$ of $S$.

Assume now that $x=\left\langle x_{1}, \ldots, x_{d}\right\rangle$ is a NCE to hypothesis $H_{r}$. For each $i \in\{1, \ldots, d\}$ with $x_{i} \neq u_{i}$ the point $x_{i}$ provides a (true or false) NCE to the current hypothesis $\left\{u_{i}, \ldots, h_{i}\right\}$ of subroutine $T B S_{n-u_{i}+1}$, or to the current hypothesis $\left\{h_{i}^{*}, \ldots, u_{i}\right\}$ of subroutine $T B S_{u_{i}}^{*}$. One updates the interval in the $i$-th dimension of the next hypothesis $H_{r+1}$ of $S$ according to the next hypothesis of $T B S_{n-u_{i}+1}$ resp. $T B S_{u_{i}}^{*}$. For those $i$ with $x_{i}=u_{i}$ one leaves the interval in the $i$-th dimension unchanged. 
By Theorem 2.1 each subroutine for learning one of the $2 d$ halfintervals encounters at most $\log n$ true NCE's. Since each NCE for algorithm $S$ provides a true NCE for at least one of the $2 d$ subroutines, $S$ gets altogether at most $2 d \log n$ NCE's. Each of these NCE's may generate false NCE's for up to $d-1$ subroutines. Hence the sum of false NCE's for all $2 d$ subroutines together is $\leq(d-1) 2 d \log n$. Thus by Theorem 2.1 the sum of all PCE's that are received by the $2 d$ subroutines is bounded by $2 d(\log n+1)+3(d-1) 2 d \log n=\left(6 d^{2}-4 d\right) \log n+2 d$. Since each PCE to algorithm $S$ (except for the first one) generates a PCE for at least one of its $2 d$ subroutines, the total number of PCE's that $S$ receives is $\leq\left(6 d^{2}-4 d\right) \log n+2 d+1$. Hence $L C(S) \leq 2 d \log n+\left(6 d^{2}-4 d\right) \log n+2 d+1=6 d^{2} \log n-2 d \log n+2 d+1$.

Remark 3.2 Peter Auer shows (1993) that $L C\left(B O X_{n}^{d}\right)=\Omega\left(d^{2} \log n / \log d\right)$. Hence the preceding algorithm is close to optimal with regard to its error bound. He also constructs an error robust variation of our learning algorithm for $B O X_{n}^{d}$, that can tolerate a certain fraction of incorrect positive and negative counterexamples for the global algorithm (for any distribution of incorrect counterexamples).

\section{An algorithm for learning the union of two boxes in the plane}

The algorithm in the preceding section was based on a solution of the credit assignment problem in which the local search procedures tolerate false negative counterexamples. It was essential for the success of this algorithm that the local search procedures never receive false positive counterexamples.

In this section we examine a more complex learning problem, in which the obvious local search procedures have to tolerate both false negative and false positive counterexamples. For any $m, n \in \mathrm{N}$ let $X_{m, n}$ be the domain

$$
\begin{aligned}
& X_{m, n}:=\{\langle i, j\rangle \mid i \in\{1, \ldots, m\} \text { and } j \in\{1, \ldots, n\}\} . \\
& \text { Set } B O X_{m, n}:=\{\{i, \ldots, j\} \times\{k, \ldots, l\} \mid 1 \leq i \leq j \leq m \\
&\text { and } 1 \leq k \leq l \leq n\} .
\end{aligned}
$$

We write $a:=\langle 1, n\rangle$ for the upper left corner and $b:=\langle m, 1\rangle$ for the lower right corner of this domain $X_{m, n}$. We consider the following concept class over the domain $X_{m, n}$ :

$$
T W O-B O X_{m, n}:=\left\{R A \cup R B \mid R A, R B \in B O X_{m, n}, a \in R A \text { and } b \in R B\right\} .
$$

Whenever we write $R A$ ( $R B$ ) in the following, we assume that $R A \in B O X_{m, n}$ and $a \in R A\left(R B \in B O X_{m, n}\right.$ and $\left.b \in R B\right)$. Note that the two components $R A$ and $R B$ of a concept in $T W O-B O X_{m, n}$ may or may not intersect.

The learning of arbitrary target concepts $R A \cup R B$ from $T W O-B O X_{m, n}$ may be viewed as a combination of 4 search procedures that determine the lengths of the sides of $R A$ and $R B$. In the same way as in the preceding section these local search procedures will receive false negative counterexamples, since it is not clear which side of $R A(R B)$ has to be shortened in order to accomodate a NCE $g \in(R A \cup R B)-C_{T}$. However these local search procedures will in general also receive false positive counterexamples, since it is not 


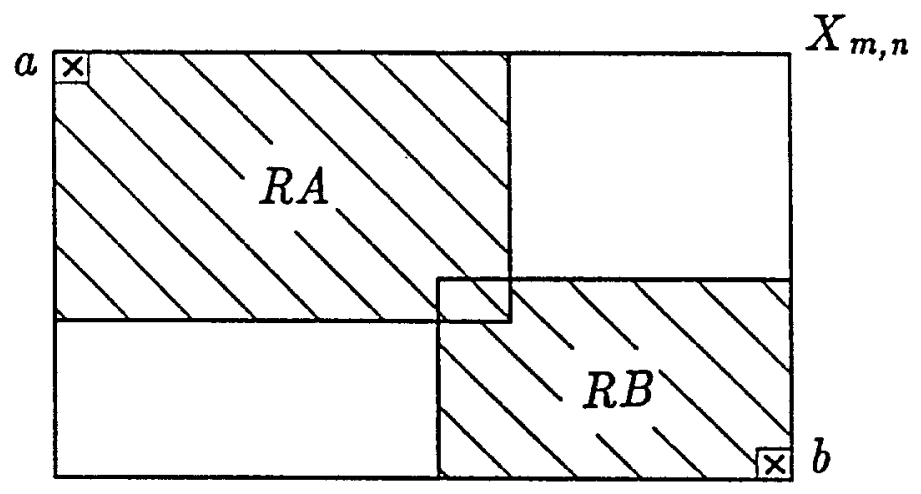

Figure 2. A typical concept $R A \cup R B$ in the concept class $T W O-B O X_{m, n}$, where $R A$ contains the upper left corner $a$ of the domain, and $R B$ contains the lower right corner $b$ of the domain.

clear whether a PCE should lie in $R A$, or in $R B$ (or in both). The following result shows that nevertheless there is an efficient learning algorithm for this learning problem.

THEOREM 4.1 LC(TWO-BOX $\left.X_{m, n}\right)=\Theta(\log (m+n))$.

Proof: It is obvious that chain $\left(T W O-B O X_{m, n}\right)=\Omega(m+n)$, where chain $(\mathcal{C})$ denotes the length of the longest chain in $\mathcal{C}$ with regard to the partial order " $\leq$ " of $\mathcal{C}$ defined by $C \leq C^{\prime} \Leftrightarrow C \subseteq C^{\prime}$. According to Maass and Turán $(1989,1992)$, one has

$$
L C\left(T W O-B O X_{m, n}\right) \geq\left\lfloor\log \left(\operatorname{chain}\left(T W O-B O X_{m, n}\right)\right)\right\rfloor .
$$

In fact, the same lower bound holds for $L C-A R B\left(T W O-B O X_{m, n}\right)$.

In order to prove the upper bound of Theorem 4.1, we first consider the following subclass of $T W O-B O X_{m, n}$ :

$$
\begin{aligned}
\mathcal{U}_{m, n}:=\{R A \cup R B \mid & R A, R B \in B O X_{m, n}, a \in R A, b \in R B, \\
& \text { and }|R A \cap R B|=1\} .
\end{aligned}
$$

We will exhibit in the proof of the main lemma (Lemma 4.3) an efficient learning algorithm $K$ for this concept class $\mathcal{U}_{m, n}$. This algorithm $K$ will employ as local search procedures the following binary search algorithm $C B S$ ("conservative binary search"), which is distinguished by the property that it never receives two successive NCE's. Although $C B S$ will also be used in a nonstandard situation (where there exists no target concept), it suffices that we analyze it here in the context of the basic learning model that was defined in section 1.

\section{Definition of the binary search algorithm $C B S$ for learning $H E A D_{n}$ :}

Assume that the environment has fixed some target concept $C_{T} \in H E A D_{n}$. At step $r$ the learner issues the hypothesis $H_{r}:=\{1, \ldots, r\} \in H E A D_{n}$. If $H_{r} \neq C_{T}$, he receives at 
step $r$ a counterexample $g_{r} \in H_{r} \Delta C_{T}$. Let $p_{s}$ be the maximum of 1 and the largest PCE received by the end of step $s$, and let $n_{s}$ be the minimum of $n+1$ and the smallest NCE received by the end of step $s$.

Set $h_{1}:=1$, and

$$
h_{s+1}:= \begin{cases}p_{s} & , \text { if } g_{s} \text { is a NCE } \\ p_{s}+\left\lfloor\frac{n_{s}-p_{s}}{2}\right\rfloor, & \text { if } g_{s} \text { is a PCE. }\end{cases}
$$

LEMMA 4.2 Consider any learning process for learning $H E A D_{n}$ (in the basic learning model) with learning algorithm $C B S$. Then there are never two successive NCE's in this learning process. Furthermore $n_{t}-p_{t} \leq \frac{n}{2^{\lfloor t / 2\rfloor}}$ for any step $t \geq 1$ with $H_{t} \neq C_{T}$.

Proof: The first claim is obvious from the construction of $C B S$.

In order to prove the second claim we show that for any $s \geq 1$ such that $g_{s}$ is a PCE and $H_{s+1} \neq C_{T}$ one has

$$
n_{s+1}-p_{s+1} \leq \frac{n_{s}-p_{s}}{2}
$$

Since $g_{s}$ is a PCE we have

$$
h_{s+1}=p_{s}+\left\lfloor\frac{n_{s}-p_{s}}{2}\right\rfloor \text {. }
$$

If $g_{s+1}$ is a NCE we have

$$
n_{s+1} \leq h_{s+1} \leq p_{s}+\frac{n_{s}-p_{s}}{2}
$$

and $p_{s+1}=p_{s}$, hence

$$
n_{s+1}-p_{s+1} \leq \frac{n_{s}-p_{s}}{2}
$$

If $g_{s+1}$ is a PCE we have

$$
p_{s+1} \geq 1+h_{s+1} \geq p_{s}+\frac{n_{s}-p_{s}}{2}
$$

and $n_{s+1}=n_{s}$, hence

$$
n_{s+1}-p_{s+1} \leq n_{s}-\left(p_{s}+\frac{n_{s}-p_{s}}{2}\right)=\frac{n_{s}-p_{s}}{2} .
$$

LEMMA 4.3 (Main lemma) $L C^{T W O-B O X_{m, n}}\left(\mathcal{U}_{m, n}\right)=O(\log (m+n))$. 


\section{Remark 4.4}

a) One has to use in Lemma 4.3 a larger hypothesis space than $\mathcal{U}_{m, n}$, since $L C\left(\mathcal{U}_{m, n}\right)=$ $\Omega(m+n)$. This lower bound can be shown with the help of an adversary strategy that gives only negative counterexamples from the "diagonal line" between $\langle 1,1\rangle$ and $\langle m, n\rangle$ in $X_{m, n}$.

b) The learning algorithm for $\mathcal{U}_{m, n}$ that is constructed in the proof of Lemma 4.3 uses actually only the hypothesis space $\left\{H \in T W O-B O X_{m, n} \mid \bar{H} \in T W O-B O X_{n, m}\right\}$, which is a proper subclass of $T W O-B O X_{m, n}$.

Proof of Lemma 4.3: In order to design an efficient learning algorithm $K$ for $\mathcal{U}_{m, n}$, we note that any concept $R A \cup R B \in \mathcal{U}_{m, n}$ with $a \in R A, b \in R B$ and $|R A \cap R B|=1$ can be uniquely characterized by the single intersection point $w=\langle i, j\rangle$ of the rectangles $R A$ and $R B$. We write $R_{w}$ for this concept $R A \cup R B$ from $\mathcal{U}_{m, n}$, and $R A_{w}, R B_{w}$ for its two components $R A, R B$.

The learning algorithm $K$ for $\mathcal{U}_{m, n}$ proceeds in a recursive manner. Assume that it has already exhibited an $m^{\prime} \times n^{\prime}$ rectangle $W \subseteq X_{m, n}$ with $w \in W$ for the target concept $R_{w} \in \mathcal{U}_{m, n}$ (initially one has $W=X_{m, n}$ ). We will use area $(W)$ (or rather: 1-area $(W)$ ) as a "measure of progress" for the learning algorithm $K$. We will not be able to guarantee that area $(W)$ can always be reduced by a fixed fraction within $O(1)$ steps of learning algorithm $K$. However we can show that there is some number $t$ (which depends on the specific learning process) such that $K$ produces in $t+2$ further steps a rectangle $\widetilde{W} \subsetneq W$ with $w \in \widetilde{W}$ and

$$
\operatorname{area}(\widetilde{W}) \leq \frac{\operatorname{area}(W)}{\max \left(2,2^{t / 4-2}\right)}
$$

Assume that $e \in X_{m, n}$ is the "centerpoint" of $W$. We first consider the case where $e$ does not lie on the perimeter of $W$ (i.e. we assume that $m^{\prime}>2$ and $n^{\prime}>2$ ). Then $K$ issues $R_{e}$ as its next hypothesis.

We will analyze separately the two cases where the learner receives a positive respectively negative counterexample $g$ to this hypothesis $R_{e}$. In each case the primary goal of the learner is to determine whether $w \in S$ or $w \in T$, where $S$ and $T$ are the two rectangles that are defined by counterexample $g$ as indicated in Figure 3. However the learner may not be able to achieve this information within a fixed number of steps. Instead, he enters a "P-phase" (in case 1), respectively an " $N$-phase" (in case 2 ). We are not able to bound the number $t$ of steps which are spent by the learner in the respective phase. However we can guarantee that at the end of such phase the learner has not only determined whether $w \in S$ or $w \in T$, but in addition he can exhibit a rectangle $\widetilde{W}{ }_{+} \underset{+}{ }$ with

$$
\operatorname{area}(\widetilde{W}) \leq \frac{\operatorname{area}(W)}{\max \left(2,2^{t / 4-2}\right)}
$$

Obviously this suffices in order to determine the target concept from $\mathcal{U}_{m, n}$ in altogether $O(\log (m+n))$ learning steps. 


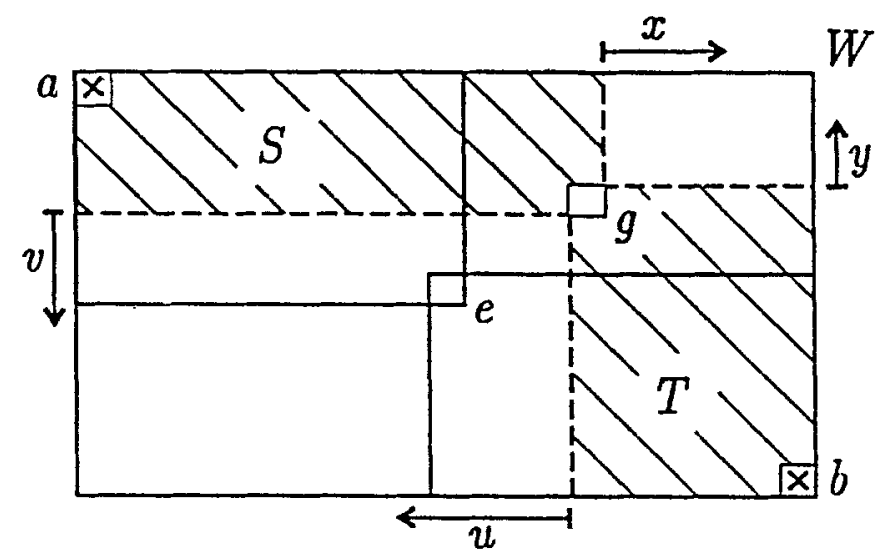

Figure 3. The intersection point $w$ of $R A$ and $R B$ is in $S \cup T$, after a positive counterexample $g$ in $W$ is received.

\section{Case 1: The learner receives a positive counterexample $g=\left\langle\boldsymbol{p}_{g}, \boldsymbol{q}_{g}\right\rangle \in \boldsymbol{R}_{w}-\boldsymbol{R}_{e}$}

We can assume without loss of generality that $g \in W$. If $g$ lies to the left (right) of $W$, we may replace it by the point on the same row in the leftmost (rightmost) column of $W$. If $g$ lies above (below) $W$, we may replace it by a point in the same column in the highest (lowest) row of $W$. Note that we have used here the assumption that $e$ does not lie on the perimeter of $W$ (i.e. $m^{\prime}>2$ and $n^{\prime}>2$ ). Furthermore it is clear that $g$ cannot lie in the area to the left and above $W$ (respectively to the right and below $W$ ), since these two areas are contained both in the target concept $R_{w}$ (because $w \in W$ ) and in the hypothesis $R_{e}$.

Let $S, T \subseteq W$ be the rectangles with $S \cap T=\{g\}$ and $R_{g} \cap W=S \cup T$, as shown in Figure 3. It is clear that $w \in S \cup T$. The algorithm $K$ issues $R_{g}$ as its next hypothesis. If $R_{g} \neq C_{T}$ it follows that $w \in S \cup T-(S \cap T)$. In order to determine whether $w \in S$ or $w \in T$, the learning algorithm $K$ enters a procedure that we call a $P$-phase. When this P-phase terminates after $t$ steps, the algorithm exhibits a rectangle $\widetilde{W}$ with $\widetilde{W} \subseteq S$ or $\widetilde{W} \subseteq T$ such that $w \in \widetilde{W}$ and $\operatorname{area}(\widetilde{W}) \leq \frac{\operatorname{area}(W)}{2^{t / 4-2}}$.

The P-phase consists of 4 concurrent binary search procedures that try to determine the values of 4 parameters $x, y, u, v$ (see Figure 3). If $w \in T$, then the values of $x$ and $v$ give the horizontal resp. vertical distance of $w$ from $g$, whereas the parameters $y$ and $u$ are undefined. If $w \in S$, then the values of $u$ and $y$ give the horizontal resp. vertical distance of $w$ from $g$, whereas the parameters $x$ and $v$ are undefined. The hypothesis of algorithm $K$ is at each step of the P-phase of the form $R A \cup R B$ with $a \in R A, S \subseteq R A, b \in R B$, and $T \subseteq R B$. The exact lengths of the sides of $R A(R B)$ are determined by the current hypotheses of the binary search procedures for $x$ and $v(u$ and $y$ ). The remainder of our analysis of case 1 is devoted to the precise description and analysis of the P-phase.

The difficulty of the P-phase is caused by the need to carry out the concurrent binary search procedures for the parameters $x, y, u, v$ without knowing whether $w \in S$ or $w \in T$, and hence without knowing which ones of $x, y, u, v$ are actually undefined. Thus we have to 
combine two "real" binary search procedures with two "dummy" binary search procedures, without knowing which are the real ones. The danger is that we may spend many learning steps exclusively for the benefit of those search procedures that later turn out to be "dummy" (i.e. they search for the values of parameters that are actually undefined). Consider for example the two search procedures for the parameters $x$ and $y$. We know that exactly one of those two parameters is undefined. If one receives a PCE $q \in R_{w}-(R A \cup R B)$ in the region above $T$ (see Figure 3 ), then this provides a PCE for both of the two binary search procedures for $x$ and for $y$ (in particular also for the "real" one among the two). However a NCE $q \in(R A \cup R B)-R_{w}$ in the region above $T$ may provide a NCE only for one of these two binary search procedures. If one has bad luck, it provides a NCE only for the one that later turns out to be "dummy", and no progress has been made at this learning step for the "real" binary search procedure among the two.

This difficulty is handled by using for the local binary search procedures the algorithm $C B S$ that was analyzed in Lemma 4.2. It may still occur then, that a NCE provides progress only for the "dummy" one among two binary search procedures $C B S$. However since no binary search procedure $C B S$ (even the "dummy" ones) may receive two NCE's in a row, this event can occur on average at most at every second step.

An exception may occur at a step where a binary search procedure that searches for an undefined parameter receives a NCE $g_{s} \leq p_{s}$ (and hence possibly two NCE's in a row). However such step (which reveals to the learner which ones of the parameters are undefined) automatically terminates the current P-phase.

We now describe in detail how the algorithm $K$ proceeds during the considered P-phase. One should keep in mind that this $P$-phase focuses its activity on the $m^{\prime} \times n^{\prime}$-rectangle $W \subseteq X_{m, n}$, but that its hypotheses are required to be from $T W O-B O X_{m, n}$. One carries out 4 concurrent binary searches with algorithm $C B S$. The first one of these is a copy of $C B S$ that searches for the value of parameter $x$, in case that $x$ is defined. More precisely: $C B S$ searches for the concept $\{0,1, \ldots, x\} \in H E A D_{\hat{n}}$ for some $\hat{n} \leq n$ (for technical reasons we take here $\{0, \ldots, \hat{n}-1\}$ as domain for $H E A D_{\hat{n}}$, instead of $\left.\{1, \ldots, \hat{n}\}\right)$. The second binary search procedure is a copy of $C B S$ that searches for the value of $y$, in case that $y$ is defined. Analogously one uses copies of $C B S$ to search for $u$ resp. $v$. Assume that so far none of these 4 copies of $C B S$ has encountered a contradiction among its counterexamples, and that $h_{x}, h_{y}, h_{u}, h_{v}$ are the endpoints of the current hypotheses $\left\{0, \ldots, h_{x}\right\},\left\{0, \ldots, h_{y}\right\},\left\{0, \ldots, h_{u}\right\},\left\{0, \ldots, h_{v}\right\}$ in the respective copies of $C B S$. Then the algorithm $K$ issues as its next hypothesis the following concept $H \in T W O-B O X_{m, n}$ :

$$
\begin{aligned}
H:= & \left\{1, \ldots, p_{g}+h_{x}\right\} \times\left\{q_{g}-h_{v}, \ldots, n\right\} \\
& \cup\left\{p_{g}-h_{u}, \ldots, m\right\} \times\left\{1, \ldots, q_{g}+h_{y}\right\} .
\end{aligned}
$$

It is obvious that $S \cup T \subseteq H$.

Let $h \in H \Delta R_{w}$ be a counterexample to this hypothesis. We will first consider the case where $h \in W$. We will analyze in the next two paragraphs the subcase where $h \in W$ is a PCE. In the subsequent third paragraph we will analyze the subcase where $h \in W$ is a NCE. After that we will turn to the analysis of the case where $h \notin W$.

If $h$ is a PCE and if $h$ lies above $T$, then one processes the two coordinates of $h$ as PCE's for the two copies of $C B S$ that search for the parameters $x$ and $y$. 
If $h \in W$ is a PCE that lies to the left of $T$, one processes the two coordinates of $h$ as PCE's for the two copies of $C B S$ that search for the parameters $u$ and $v$.

If $h \in W$ is a NCE with $h \notin S \cup T$ and $h$ lies above $T$, then the two coordinates of $h$ provide a NCE for at least one of the two copies of $C B S$ that search for $x$ and $y$. (Since $h$ cannot be guaranteed to provide a NCE for both copies of $C B S$, it may potentially only provide a NCE for the "dummy" copy of $C B S$ ). If $h \in W$ is a NCE with $h \notin S \cup T$ and $h$ lies to the left of $T$, then the two coordinates of $h$ provide a NCE for at least one of the two copies of $C B S$ that search for $u$ and $v$.

If $h \in W$ is a NCE with $h \in S \cup T$ then it terminates the current P-phase. If $h \in T$, then it is proven that $w \in T$, and that the parameters $y$ and $u$ are undefined. The current P-phase also ends if in any of the preceding cases at least one of the 4 copies of $C B S$ receives a $\mathrm{CE}$ that contradicts another $\mathrm{CE}$ that it had received at an earlier step. Assume for example that the copy of $C B S$ that searches for $x$ receives a PCE (NCE) that contradicts an earlier NCE (PCE). This implies that the parameter $x$ is undefined. Hence one has $w \in S$, which implies that the parameter $v$ is also undefined.

Finally we consider the case where the counterexample $h \in H \Delta R_{w}$ does not lie in $W$. If $h$ is a PCE to the right of $W$, then it implies that $w \in S$. Hence this counterexample terminates the current P-phase. If $h$ is a NCE to the right of $W$, it provides a NCE for the binary search for $y$ (but no CE for the binary search for $x$ ). The cases where $h$ lies above, below, or left of $W$ are handled analogously.

It remains to be shown that in each possible case where the current P-phase is terminated, one can not only decide whether $w \in S$ or $w \in T$, but one can also exhibit an axis-parallel rectangle $\widetilde{W}$ with $\widetilde{W} \subseteq S$ or $\widetilde{W} \subseteq T, w \in \widetilde{W}$, and area $(\widetilde{W}) \leq \frac{\operatorname{area}(W)}{\max \left(2,2^{t / 4-2}\right)}$, where $t$ is the number of counterexamples that have been received during the current $P$-phase.

Each PCE $h$ that is received before the end of the P-phase provides a PCE for both of the binary search procedures for $x$ and $y$, or for both of the binary search procedures for $u$ and $v$. Each NCE provides a NCE for at least one of the binary search procedures for $x$ and $y$, or for at least one of the binary search procedures for $u$ and $v$. Since none of these 4 copies of procedure $C B S$ (not even those that search for undefined parameters) can receive two successive NCE's (except at the last step of this P-phase), at least $t^{\prime}:=\frac{t}{2}-3$ of the $t$ counterexamples of this P-phase provide CE's for one of the two copies of $C B S$ which search for parameters that are actually defined. By Lemma 4.2 at least $\left\lfloor\frac{t^{\prime}}{2}\right\rfloor$ of them reduce one of the two dimensions of $\widetilde{W}$ by at least $50 \%$, starting with $S$ resp. $T$. Since area $(S)$, $\operatorname{area}(T) \leq \frac{\operatorname{area}(W)}{2}$, one has

$$
\operatorname{area}(\widetilde{W}) \leq \frac{\operatorname{area}(W)}{2^{1+\left\lfloor\frac{1}{2}(t / 2-3)\right\rfloor}} \leq \frac{\operatorname{area}(W)}{2^{t / 4-2}}
$$

Finally we observe that any P-phase terminates at the latest after $O(\log (m+n))$ step, since each single one of the 4 procedures $C B S$ can receive at most $2 \log (m+n)$ counterexamples without running into a contradiction. 


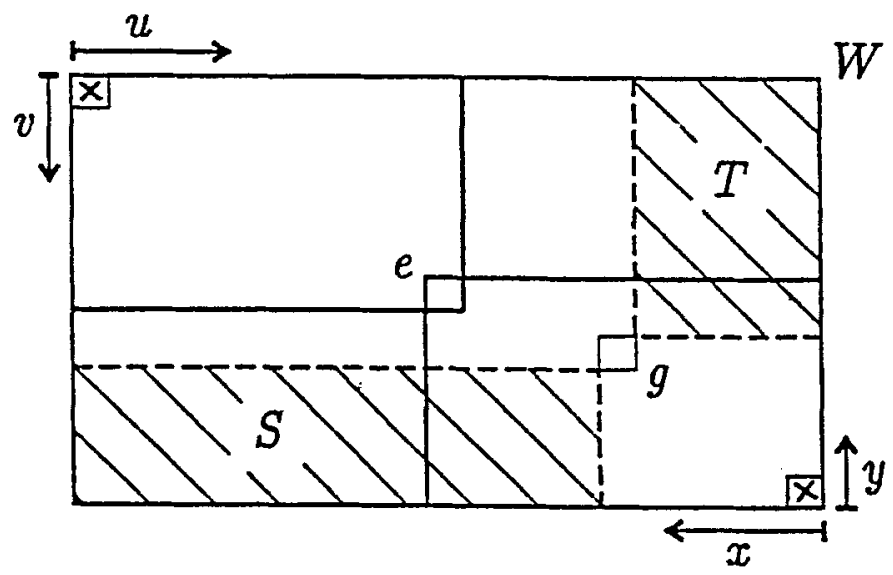

Figure 4. The intersection point $w$ of $R A$ and $R B$ is in $S \cup T$, after a negative counterexample $g$ in $W$ is received.

Case 2: The learner receives a negative counterexample $g=\left\langle p_{g}, q_{g}\right\rangle \in \boldsymbol{R}_{e}-\boldsymbol{R}_{w}$.

If $g \notin W$ then we can immediately exhibit a rectangle $\widetilde{W} \subseteq W$ with $w \in \widetilde{W}$ and $\operatorname{area}(\widetilde{W}) \leq \frac{\operatorname{area}(W)}{2}$. Since $g \in R_{e}=R A \cup R B$, we know that $g$ lies either in the rectangle $R A$ of $R_{e}$ that contains the upper left corner $a$ of the domain $X_{m, n}$, or $g$ lies in the rectangle $R B$ that contains the lower right corner $b$ of the domain $X_{m, n}$. If $g \in R A$ and $g$ lies above $W$, then $g \notin R_{w}$ implies that $w$ lies in the left half of $W$. If $g \in R A$ and $g$ lies left of $W$, then $g \notin R_{w}$ implies that $w$ lies in the upper half of $W$. Hence in either case we can exhibit a rectangle $\widetilde{W}$ with $w \in \widetilde{W}$ and area $(\widetilde{W}) \subseteq \frac{\operatorname{area}(W)}{2}$. The argument for $g \in R B$ is analogous.

We now assume that $g \in W$.

It is then clear that $w \in S \cup T$ for the rectangles $S, T$ that are defined by $g$ as indicated in Figure 3. In order to determine whether $w \in S$ or $w \in T$ the algorithm then enters an $N$-phase. An N-phase consists of 4 concurrent binary searches that determine the values of 4 parameters $x, y, u, v$. If $w \in T$, then the value of $x$ is the horizontal distance of $w$ from the rightmost column of $W$, the value of $v$ is the vertical distance of $w$ from the top row of $W$, and the parameters $y$ and $u$ are undefined. If $w \in S$, then the value of $y$ is the vertical distance of $w$ from the bottom row of $W, u$ is the horizontal distance of $w$ from the leftmost column of $W$, and the parameters $x$ and $v$ are undefined. Each hypothesis during the $\mathrm{N}$-phase is the union of two rectangles $R A$ and $R B . R A$ is contained in $\left\{1, \ldots, p_{g}-1\right\} \times\left\{q_{g}+1, \ldots, n\right\}$, and the lengths of its sides are determined by the binary search procedures for $u$ and $v$. Analogously $R B$ is contained in $\left\{p_{g}+1, \ldots, m\right\} \times\left\{1, \ldots, q_{g}-1\right\}$, and the lengths of its sides are determined by the binary search procedures for $x$ and $y$. In order to verify Remark 4.4 (b) we note that $\overline{R A \cup R B} \in T W O-B O X_{n, m}$ since no row and no column contains points from both $R A$ and $R B$. 
In contrast to the situation in a $\mathrm{P}$-phase, a PCE $h$ to hypothesis $R A \cup R B$ may yield a PCE only for one of the search procedures for $u$ and $v$ (if $h$ lies above $S$ ), or only for one of the search procedures for $x$ and $y$ (if $h$ lies below T). On the other hand, a NCE to hypothesis $R A \cup R B$ provides a NCE either for both search procedures for $u$ and $v$, or for both search procedures for $x$ and $y$. Hence one uses here as binary search procedures for $x, y, u, v$ a dual version $C B S^{\prime}$ of $C B S$, for which no two successive PCE's can occur.

Note that any PCE $h \in\left\{1, \ldots, p_{g}\right\} \times\left\{1, \ldots, q_{g}\right\} \cup\left\{p_{g}, \ldots, m\right\} \times\left\{q_{g}, \ldots, n\right\}$ reveals whether $w \in S$ or $w \in T$, and it will automatically terminate this $\mathrm{N}$-phase. Similarly a NCE outside of $W$ decides whether $w \in S$ or $w \in T$, and it also terminates this N-phase. Apart from these cases, the $\mathrm{N}$-phase is also terminated by any counterexample that provides a contradiction to an earlier counterexample for any of the 4 copies of the binary search procedure $C B S^{\prime}$. The rest of the analysis of the $\mathrm{N}$-phase is analogous to that of the P-phase.

Finally we have to comment on the case where the algorithm $K$ cannot continue its recursion with a P-phase or an N-phase, because it has already narrowed down the location of $w$ to an $m^{\prime} \times n^{\prime}$ rectangle $W \subseteq X_{m, n}$ with $m^{\prime} \leq 2$ or $n^{\prime} \leq 2$. Assume for example that $m^{\prime}=2$. Then the algorithm $K$ carries out for both columns in $W$ a straightforward binary search for $w$. This is possible, because for the binary search in the "correct" column it can interpret each counterexample without ambiguity.

This completes the proof of Lemma 4.3.

With the help of the preceding main lemma we are now able to prove Theorem 4.1. Besides the cornerpoints $a=\langle 1, n\rangle$ and $b=\langle m, 1\rangle$ we will also distinguish the other two cornerpoints $c=\langle 1,1\rangle$ and $d=\langle m, n\rangle$ of the domain $X_{m, n}$. For many $C \in T W O-B O X_{m, n}$ the complement $\bar{C}:=X_{m, n}-C$ may be viewed as element of $B O X_{m, n}, B O X_{n, m}$ or $T W O-B O X_{n, m}$. In order to consider $\bar{C}$ as element of $T W O-B O X_{n, m}$ one "turns the domain by $90^{\circ}$ ", i.e. one identifies $X_{m, n}$ with $X_{n, m}, a$ with $c$, and $b$ with $d$ (see Figure 4). This duality is frequently exploited in the following in order to discuss subroutines that aim at learning $\bar{C}_{T}$ instead of $C_{T}$. This makes sense for those cases where $\bar{C}_{T}$ has a simpler structure than $C_{T}$. Note however that one has to be careful when one exploits this duality, since there are $C \in T W O-B O X_{m, n}$ for which $\bar{C}$ can not be interpreted as a union of one or two rectangles (e.g. consider $C=\{1, \ldots, m-1\} \times\{n\} \cup\{2, \ldots, m\} \times\{1\}$ ).

The learning algorithm $L$ for $T W O-B O X_{m, n}$ proceeds in 4 phases. The first hypothesis of the first phase is the set $X_{m, n}$. If $X_{m, n} \neq C_{T}$, then one receives a NCE. It is then clear that $c \notin C_{T}$ or $d \notin C_{T}$. In order to eliminate the case where $\{c, d\} \cap C_{T} \neq \emptyset$, one uses as a subroutine some learning algorithm $A$ for $B O X_{m, n}$ that is guaranteed to find any $C \in B O X_{m, n}$ in $O(\log (m+n))$ steps, using hypotheses from $B O X_{m, n}$ (see section 3, or Maass \& Turán (1994)). One first executes this learning algorithm $A$ in order to find $\bar{C}_{T}$ under the assumption that $c \in \bar{C}_{T}$ and $d \in C_{T}$ (hence $\bar{C}_{T} \in B O X_{m, n}$ ) by inverting the "sign" of each example and by replacing each hypothesis $H$ of $A$ by its complement $\bar{H}$ (note that $\bar{H} \in T W O-B O X_{m, n}$ for any $H \in B O X_{m, n}$ with $c \in H$ ). In this way one finds $C_{T}$ in $O(\log (m+n))$ steps if $c \in \bar{C}_{T}$ and $d \in C_{T}$. If this attempt is not successful, one executes $A$ again in order to find $\bar{C}_{T}$, but this time under the assumption that $d \in \bar{C}_{T}$ and $c \in C_{T}$. If this attempt is also not successful, one has proven that $c \in \bar{C}_{T}$ and $d \in \bar{C}_{T}$. 
During its second phase the algorithm $L$ checks whether $\bar{C}_{T} \in \mathcal{U}_{n, m}$. For this purpose it executes the algorithm $K$ from the proof of Lemma 4.3 for $O(\log (m+n))$ steps over the domain $X_{n, m}$ in order to learn the complement of $C_{T}$. Hence the sign of each example is inverted, and each hypothesis $H$ of algorithm $K$ is replaced by its complement $\bar{H}$. Note that according to Remark 4.4 (b) this algorithm $K$ for $\mathcal{U}_{n, m}$ uses only hypotheses $H$ such that $\bar{H} \in T W O-B O X_{m, n}$. Hence $\bar{H}$ is a permissible hypothesis for algorithm $L$. If this simulation of $K$ fails to identify $\bar{C}_{T}$ within its alloted time, we know that $\bar{C}_{T} \notin \mathcal{U}_{n, m}$. Furthermore the sample $S$ that has been assembled by this time has the property that no $C \in T W O-B O X_{m, n}$ with $\bar{C} \in \mathcal{U}_{n, m}$ is consistent with $\mathrm{S}$. (We refer to a set of positive and/or negative examples for $C_{T}$ as a sample for $C_{T}$.)

During its third phase the learning algorithm $L$ checks whether $C_{T}$ is of the form $R A \cup R B$ with $R A \cap R B \neq \emptyset$. One uses here the following simple structural result.

LEMMA 4.5 Assume that $S$ is a sample that is consistent with some $C=R A \cup R B \in$ $T W O-B O X_{m, n}$ such that $c, d \notin C$ and $R A \cap R B \neq \emptyset$. Furthermore assume that $S$ is not consistent with any $C \in T W O-B O X_{m, n}$ such that $\bar{C} \in \mathcal{U}_{n, m}$.

Then there do not exist among those concepts that are consistent with $S$ for $i=1,2$ concepts $C_{i}=R A_{i} \cup R B_{i}$ with $R A_{i} \cap R B_{i} \neq \emptyset$ and $\bar{C}_{i}$ of the form $R C_{i} \cup R D_{i}$ with $R C_{i}, R D_{i} \in B O X_{m, n}, c \in R C_{i}, d \in R D_{i}$, such that $g \in R C_{1} \cap R D_{2}$ for some negative example $g$ in $S$.

In other words: the assignment of negative examples in $S$ to the two rectangles of $\bar{C}$ is unique (for concepts $C=R A \cup R B$ with $R A \cap R B \neq \emptyset, c, d \notin C$ ).

Proof of Lemma 4.5: Assume for a contradiction that there exist such concepts $C_{1}, C_{2}$ and such negative example $g \in R C_{1} \cup R D_{2}$ in $S$. Then there are two different rows (resp. columns) $r_{1}, r_{2}$ in the domain $X_{m, n}$ such that $r_{1} \subseteq C_{1}, r_{2} \subseteq C_{2}$, and $g$ lies strictly between $r_{1}$ and $r_{2}$. Without loss of generality we assume that $r_{1}, r_{2}$ are rows with $r_{1}$ above $r_{2}$ (see Figure 5).

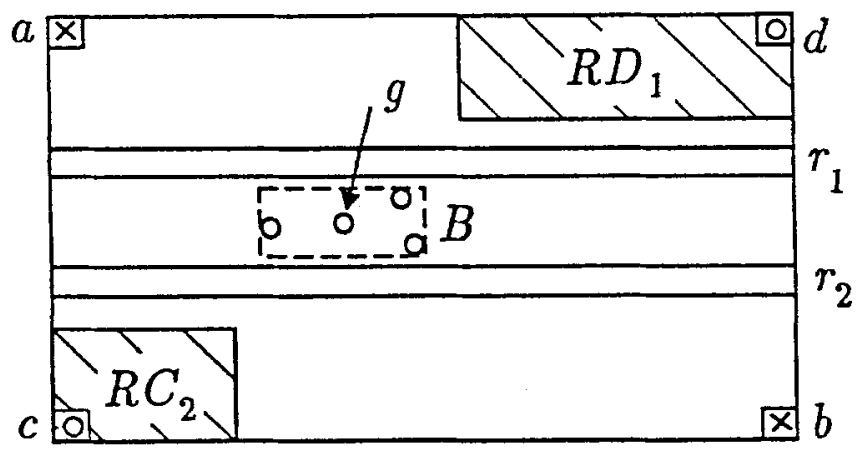

Figure 5. Negative counterexamples in the sample $S$ are contained in $B$, or lie above $r_{1}$ and to the right of the rightmost column of $B$, or lie below $r_{2}$ and to the left of the leftmost column of $B$. 


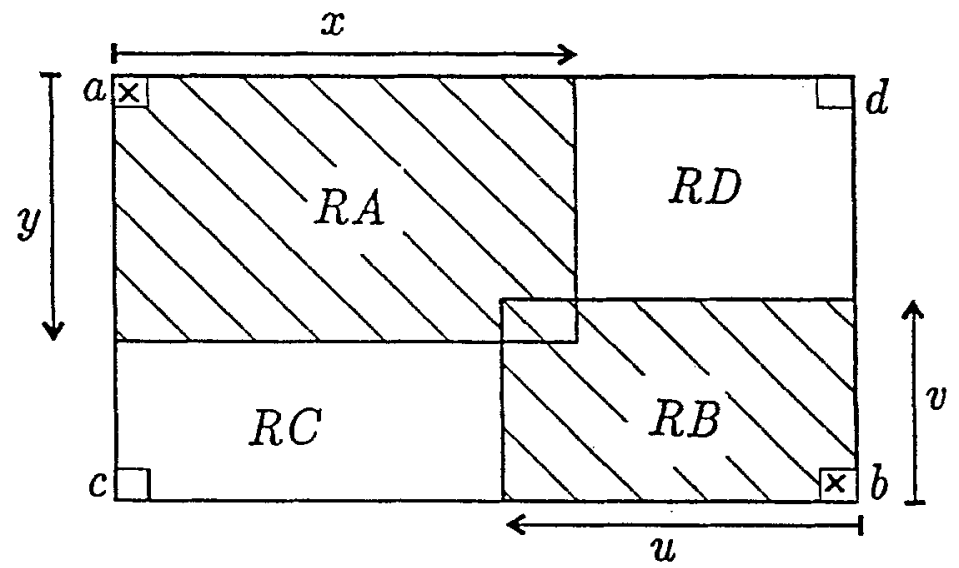

Figure 6. Searching the boundaries of $R A$ and $R B$ with four procedures which "tolerate" false positive counterexamples.

Let $B$ be the smallest axis parallel rectangle that contains all negative examples in $S$ which lie between $r_{1}$ and $r_{2}$. Note that $g \in B$. Since $C_{1}$ is consistent with $S$, all negative examples in $S$ that lie above $r_{1}$ are contained in $R D_{1}$, hence they lie to the right of the rightmost column of $B$. Since $C_{2}$ is consistent with $S$, all negative examples in $S$ that lie below $r_{2}$ are contained in $R C_{2}$, hence they lie to the left of the leftmost column of $B$. In particular all negative examples in $S$ are contained in $B \cup R C_{1} \cup R D_{2}$.

Since $B \subseteq R C_{1} \cap R D_{2}$ we can define rectangles $\overparen{R C} \subseteq R C_{1}$ and $\overparen{R D} \subseteq R D_{2}$ with $c \in \widehat{R C}$ and $d \in \overparen{R D}$ such that $\overparen{R C}$ and $\overparen{R D}$ intersect exactly at the top right corner of $B$. Hence $\widetilde{R C} \cup \widetilde{R D} \in \mathcal{U}_{n, m}$. By construction we have $B \cup R C_{1} \cup R D_{2} \subseteq \widehat{R C} \cup \widehat{R D}$, hence $\widetilde{R C} \cup \widetilde{R D}$ contains all negative examples in $S$. Furthermore $\widetilde{R C} \cup \widetilde{R D}$ does not contain any positive examples in $S$, since $R C_{1} \cup R D_{2}$ does not contain any positive example in $S$ and $\overparen{R C} \cup \widehat{R D} \subseteq R C_{1} \cup R D_{2}$. Hence the complement of $\widehat{R C} \cup \widehat{R D}$ is a concept $C \in T W O-B O X_{m, n}$ that is consistent with $S$ and which satisfies $\bar{C}=\widetilde{R C} \cup \widetilde{R D} \in \mathcal{U}_{n, m}$. However such concept $C$ does not exist by the assumption of Lemma 4.5.

Remark 4.6 We would like to point out that the unique partition of negative examples in $S$ (that exists by Lemma 4.5) can be computed in an efficient manner. One can assume without loss of generality that $R C$ and $R D$ are "spanned" by $c$ (resp. $d$ ) and the negative examples in $S$ that are assigned to them. Hence it suffices to cycle through all pairs $p_{1}, p_{2}$ of negative examples in $S$ and check whether the rectangle that is spanned by $\left\{c, p_{1}, p_{2}\right\}$ is a feasible solution for $R C$.

The strategy of $L$ during its third phase is the following. It employs 4 concurrent copies of the dual version $T B S^{\prime}$ of the error tolerant binary search procedure $T B S$ from section 2 . $T B S^{\prime}$ "tolerates" false PCE's (in a sense analogous to Theorem 2.1), as long as it receives only true NCE's. If $C_{T}=R A \cup R B$ with $R A \cap R B \neq \emptyset$ then these 4 copies of $T B S^{\prime}$ will find the lengths $x, y, u, v$ of the sides of the rectangles $R A, R B$ (see Figure 6). 
Let $\widetilde{S}$ be any extension of the so far collected set $S$ of examples by further examples for $C_{T}$. Then $\widetilde{S}$ satisfies the assumptions of Lemma 4.5 (provided that $C_{T}$ is of the form $R A \cup R B$ with $R A \cap R B \neq \emptyset$ ). Hence one can uniquely (and efficiently) assign any negative example in $\widetilde{S}$ to one of the rectangles $R C, R D \in B O X_{m, n}$ with $R C \cup R D=\bar{C}_{T}, c \in R C, d \in R D$. Obviously any negative example that has been assigned to $R C(R D)$ provides true negative examples for the binary search procedures for $u$ and $y$ ( $x$ and $v$ ). This is the reason why false NCE's can be avoided in the 4 concurrent binary searches of this phase. The hypothesis $H$ of $L$ during this phase will always be of the form

$$
H:=\left\{1, \ldots, h_{x}\right\} \times\left\{n-h_{y}+1, \ldots, n\right\} \cup\left\{m-h_{u}+1, \ldots, m\right\} \times\left\{1, \ldots, h_{v}\right\},
$$

where $h_{x}, h_{y}, h_{u}, h_{v}$ are the endpoints of the hypotheses for the associated binary search procedures $T B S^{\prime}$. Whenever one receives a NCE $g \in H-C_{T}$, one determines the unique assignment of $g$ to $R C$ or $R D$ (among all $C \in T W O-B O X_{m, n}$ that are consistent with all examples received so far, and which satisfy $C_{T}=R A \cup R B$ with $R A \cap R B \neq \emptyset$ ). Hence the coordinates of $g$ provide true NCE's for one or two copies of $T B S^{\prime}$ (and no false NCE for any copy of $T B S^{\prime}$ ). On the other hand the coordinates of a PCE $g \in C_{T}-H$ are interpreted as positive examples for all copies of $T B S^{\prime}$. Hence $g$ provides a true PCE for at least one copy of $T B S^{\prime}$, and false PCE's for up to 3 copies of $T B S^{\prime}$.

An analogous version of Theorem 2.1 for $T B S^{\prime}$ implies that all 4 copies of $T B S^{\prime}$ together can receive at most $4 \log (m+n)$ true PCE's. Hence at most $12 \log (m+n)$ false PCE's, and consequently at most $1+37 \log (m+n)$ NCE'S can be received altogether by the 4 copies of $T B S^{\prime}$ that are employed by $L$ during this third phase. If in fact $C_{T}=R A \cup R B$ with $R A \cap R B \neq \emptyset$, then $L$ will identify $C_{T}$ during this phase. Of course we terminate this phase if it has not lead to the identification of $C_{T}$ within its alloted time, or if it runs into some contradiction (which can only arise if $C_{T}$ is not of the conjectured form).

If the third phase of $L$ has not succeeded in identifying $C_{T}$, one may conclude that the set $S$ of examples that has been collected up to this point is not consistent with any $C=R A \cup R B \in T W O-B O X_{m, n}$ such that $R A \cap R B \neq \emptyset$. Hence we can apply the following simple structural result.

LEMMA 4.7 Assume that $S$ is a sample that is consistent with some $C \in T W O-$ $B O X_{m, n}$. Furthermore assume that $S$ is not consistent with any $C=R A \cup R B \in$ $T W O-B O X_{m, n}$ with $R A \cap R B \neq \emptyset$.

Then there do not exist two concepts $C_{1}, C_{2} \in T W O-B O X_{m, n}$ that are both consistent with $S$, and where $C_{i}=R A_{i} \cup R B_{i}, a \in R A_{i}, b \in R B_{i}$, and the rectangles $R A_{i}$ and $R B_{i}$ are separated by horizontal lines for $i=1,2$, such that $g \in R A_{2} \cap R B_{1}$ for some positive example $g$ in $S$.

In other words: the assignment of positive examples in $S$ to $R A, R B$ is unique among all consistent concepts $C=R A \cup R B$ whose components $R A, R B$ are separated by horizontal lines.

The analogous result holds for concepts whose components are separated by vertical lines. 
Proof of Lemma 4.7: Assume for a contradiction that there exist such concepts $C_{1}=$ $R A_{1} \cup R B_{1}$ and $C_{2}=R A_{2} \cup R B_{2}$ (whose components are separated by two horizontal lines) and some positive example $g$ in $S$ with $g \in R A_{2} \cap R B_{1}$. Then $R A_{2} \cup R B_{1}$ is a concept in $T W O-B O X_{m, n}$ that is consistent with $S$, and whose components $R A_{2}, R B_{1}$ have a nonempty intersection. However such concept does not exist by the assumption of Lemma 4.7.

During its fourth phase the algorithm $L$ first checks whether $C_{T}=R A \cup R B$ for rectangles $R A, R B$ that are separated by some horizontal line. In the same way as in phase 3 it employs 4 concurrent binary search procedures that search for the lengths $x, y, u, v$ of the sides of $R A, R B$ (see Figure 4). Each hypothesis $H$ of $L$ is constructed from the current hypotheses of the 4 binary search procedures in the same way as in phase 3 . However during this phase we use for these procedures instead of $T B S^{\prime}$ the original error tolerant binary search procedure TBS from section 2 (which "tolerates" false NCE's but no false PCE's).

Whenever a PCE $g \in C_{T}-H$ is given to $L$, it can decide with the help of Lemma 4.7 whether $g \in R A$ or $g \in R B$ (provided that $C_{T}=R A \cup R B$ for rectangles $R A, R B$ that are separated by a horizontal line). Hence it can give the coordinates of $g$ as true positive examples to those copies of $T B S$ that search for $x$ and $y$ (if $g \in R A$ ), resp. to those copies of $T B S$ that search for $u$ and $v$ (if $g \in R B$ ). Since $g \notin H$, it will provide a true PCE for at least one of these 4 copies of $T B S$ (but no false PCE for any of them).

Any NCE $g \in H-C_{T}$ for the hypothesis $H$ of $L$ provides a true NCE for at least one of the 4 copies of $T B S$ (and false NCE's for up to three copies of $T B S$ ).

If $C_{T}$ consists in fact of two rectangles that are separated by some horizontal line, $L$ will identify $C_{T}$ during this phase in at most $1+37 \log (m+n)$ steps (by Theorem 2.1).

If $L$ does not identify $C_{T}$ in this way, we know that the components $R A, R B$ of $C_{T}$ are separated by a vertical line. Hence it suffices to repeat the preceding process for the case of vertical separations.

Each phase of $L$ takes at most $O(\log (m+n))$ steps. Hence the proof of Theorem 4.1 is now complete.

Remark 4.8 With regard to the general structure of the proof of Theorem 4.1 we would like to point out that it is necessary to apply the main lemma (Lemma 4.3) to the complements of the concepts $C \in T W O-B O X_{m, n}$, rather than to the concepts themselves. This arises from a rather subtle aspect of the third phase of the algorithm. This third phase relies on the structural result of Lemma 4.5, which does not have an appropriate "dual version" (with $C$ and $\bar{C}$ interchanged). A source of this asymmetry is the fact that the two rectangles $R C, R D$ which form the complement of some $C=R A \cup R B \in T W O-B O X_{m, n}$ with $R A \cap R B \neq \emptyset$ have no common row or column. However the two components $R A, R B$ of some $C=R A \cup R B \in T W O-B O X_{m, n}$ with $R A \cap R B=\emptyset$ may very well have a common row or column.

Remark 4.9 One can use the algorithm $L$ from Theorem 4.1 as a subroutine in order to get an efficient learning algorithm for the concept class $\mathcal{U}-2-B O X_{n}^{2}:=\left\{C_{1} \cup C_{2} \mid C_{1}, C_{2} \in\right.$ $\left.B O X_{n}^{2}\right\}$. One starts each learning process by executing a learning algorithm for $B O X_{n}^{2}$, untill one has collected a sample $S$ that is not consistent with any $C \in B O X_{n}^{2}$. It is easy to 
show that any such sample $S$ contains two positive examples $a, b$ and a negative example $q$ s.t. $q$ lies in the rectangle $R$ that is spanned by $a$ and $b$. It is then clear that $a$ and $b$ lie in different components of $C_{T} \in \mathcal{U}-2-B O X_{n}^{2}$. This implies that $C_{T} \cap R \in T W O-B O X_{\bar{m}, \tilde{n}}$ for $X_{\tilde{m}, \tilde{n}}:=R$. Hence one can apply the learning algorithm $L$ from Theorem 4.1 over the domain $R$ in order to learn $C_{T} \cap R$, and separate learning algorithms for $B O X$ (resp. $T W O-B O X)$ for other parts of the domain. At each step the hypothesis of the resulting learning algorithm for $\mathcal{U}-2-B O X_{n}^{2}$ is the union of the hypotheses that result from the subroutines for various parts of the domain. One gets in this way a learning algorithm that is guaranteed to find $C_{T}$ in $O(\log n)$ step, but whose hypotheses consist of more than 2 rectangles.

\section{Open problems}

One challenging open problem is posed by the gap between our upper bound $O\left(d^{2} \log n\right)$ and Auer's (1993) lower bound of $\Omega\left(d^{2} \log n / \log d\right)$ for $L C\left(B O X_{n}^{d}\right)$.

Furthermore most questions concerning the on-line learning complexity of the concept class

$$
\mathcal{U}-k-B O X_{n}^{d}:=\left\{C_{1} \cup \cdots \cup C_{k} \mid C_{1}, \ldots, C_{k} \in B O X_{n}^{d}\right\}
$$

are still open. In particular, it is open whether $L C\left(\mathcal{U}-k-B O X_{n}^{d}\right)=O($ poly $(\log n, d))$ for constant $k \geq 2$. Even for the special case $k=d=2$ it is not known whether $L C\left(\mathcal{U}-2-B O X_{n}^{2}\right)=O(\log n)$ (although there is a positive result with a slightly larger hypothesis space; see Remark 4.9). Very recently Chen (1993) has shown that $L C\left(\mathcal{U}-2-B O X_{n}^{2}\right)=O\left(\log ^{2} n\right)$.

\section{Acknowledgment}

We would like to thank David Haussler for drawing our attention to the problem of efficient on-line learning of rectangles in arbitrary dimensions. Furthermore we are grateful to two anonymous referees for their helpful comments.

\section{References}

Angluin, D. (1988). Queries and concept learning. Machine Learning, 2, 319-342.

Auer, P. (1993). On-line learning of rectangles in noisy environments. Proc. of the 6th Annual ACM Conference on Computational Learning Theory (pp. 253-261). New York: ACM-Press.

Borgstrom, R.S. \& Kosaraju, S.R. (1993). Comparison-based search in the presence of errors. Proc. of the 25th Annual ACM Symposium on the Theory of Computing (pp. 130-136). New York: ACM-Press.

Chen, Z. (1993). Learning unions of two rectangles in the plane with equivalence queries. Proc. of the 6th Annual ACM Conference on Computational Learning Theory (pp. 243-252). New York: ACM-Press.

Cohen, P.R. \& Feigenbaum, E.A. (1982). The Handbook of Artificial Intelligence, vol. 3, Los Altos, CA: William Kaufmann. 
Dhagat, A., Gacs, P., \& Winkler, P. (1992). On playing "Twenty Questions" with a liar. Proc. of the 3rd Annual ACM-SIAM Symposium on Discrete Algorithms (pp. 16-22). New York: ACM-Press.

Haussler, D. (1989). Personal Communication.

Littlestone, N. (1988). Learning quickly when irrelevant attributes abound: a new linear threshold algorithm. Machine Learning, 2, 285-318.

Maass, W., \& Turán, G. (1989). On the complexity of learning from counterexamples (extended abstract). Proc of the 30th Annual I.E.E.E. Symposium on Foundations of Computer Science (pp. 262-267). Los Alamitos, CA: IEEE Computer Society Press.

Maass, W., \& Turán, G. (1994). Algorithms and lower bounds for on-line learning of geometrical concepts. Machine Learning, 14, 251-269.

Maass, W., \& Turán, G. (1992). Lower bound methods and separation results for on-line learning models. Machine Learning, 9, 107-145.

Pitt, L. \& Valiant, L.G. (1988). Computational limitations on learning from examples. J. of the ACM, 35, $965-984$.

Soare, R.I. (1987) Recursively Enumerable Sets and Degrees. Berlin: Springer.

Valiant, L.G. (1984). A theory of the learnable. Comm. of the ACM, 27, 1134-1142.

Received October 30, 1992

Accepted October 29, 1993

Final Manuscript November 22, 1993 